\title{
Chip-based nanoscopy: towards integration and high-throughput imaging
}

David Coucheron, Øystein Helle, Christina Øie, Firehun Dullo, Balpreet Ahluwalia

David A. Coucheron, Øystein I. Helle, Christina I. Øie, Firehun T Dullo, Balpreet S. Ahluwalia, "Chip-based nanoscopy: towards integration and highthroughput imaging," Proc. SPIE 10350, Nanoimaging and Nanospectroscopy V, 103500W (14 December 2017); doi: 10.1117/12.2273902

SPIE Event: SPIE Nanoscience + Engineering, 2017, San Diego, California, United States 


\title{
Chip Based Nanoscopy: Towards Integration and High-throughput Imaging
}

\author{
David A. Coucheron*1, Øystein I. Helle ${ }^{1}$, Cristina I. Øie ${ }^{1}$, Firehun T. Dullo ${ }^{1}$, Balpreet S. Ahluwalia ${ }^{1}$ \\ ${ }^{1}$ UiT - The Arctic University of Norway, Department of Physics and Technology, 9037 Tromsø, \\ Norway. \\ *david.a.coucheron@uit.no
}

\begin{abstract}
Super-resolution optical microscopy, commonly referred to as optical nanoscopy, has enabled imaging of biological samples with a resolution that was only achievable previously using electron microscopy. Optical nanoscopy is a rapidly growing field, with several different techniques and implementations that overcome the diffraction limit of light. However, the common nanoscope continues to be a rather complex, expensive and bulky instrument. Direct stochastic optical reconstruction microscopy (dSTORM) imaging was recently demonstrated using a waveguide platform for excitation in combination with a simple microscope for imaging. High refractive index waveguide materials have a high intensity evanescent field stretching around 100-200 nm outside the guiding material, which is ideally suited for total internal reflection fluorescence (TIRF) excitation over large areas. We demonstrate dSTORM imaging of the plasma membrane of liver sinusoidal endothelial cells (LSECs) and trophoblasts (HTR-8 cells) using waveguide excitation, with resolution down to around $70 \mathrm{~nm}$. Additionally, we present TIRF imaging of LSEC micro-tubules over a $500 \mu \mathrm{m}$ x 500 $\mu \mathrm{m}$ area, laying the foundation for large field of view (f-o-v) nanoscopy.
\end{abstract}

Keywords: Waveguide, dSTORM, TIRF, LSEC, HTR-8, super-resolution optical microscopy

\section{INTRODUCTION}

The fluorescence microscope is an indispensable tool in biological research, and has contributed to many important discoveries ${ }^{1}$. However, the spatial resolution of the technique has long been limited by the diffraction limit of light. As light from a point emitter is focused by a lens to form an image, it will actually focus into an airy disk of lateral size approximately half the wavelength of the light (ca. 250-300 nm) ${ }^{2}$. The diffraction limit restricts the optical resolution of imaging techniques, as two closely placed overlapping airy disks will be unresolvable, giving rise to the resolution limit. Overcoming the diffraction limit is of great importance, as many biological processes and structures are smaller than the diffraction limit, and are therefore unresolvable by optical microscopy.

Research into super-resolution optical microscopy started a couple of decades ago and has led to several different techniques, each with different advantages and disadvantages. In 1994, Hell et al. proposed a theoretical basis for overcoming the resolution limit in optical microscopy ${ }^{3}$. In the following years, Hell and coworkers demonstrated and advanced the technique called saturated emission depletion microscopy (STED). In 2006, super-resolution optical microscopy emerged as a research field, with three similar fluorescence based techniques introduced almost simultaneously, commonly termed single molecule localization (SLM) microscopy. The techniques are fluorescence photoactivation localization microscopy (FPALM) ${ }^{4}$, photoactivated localization microscopy (PALM) ${ }^{5}$, and stochastic optical reconstruction microscopy (STORM) ${ }^{6}$. Two years later, a more versatile and general approach to STORM was introduced, termed direct-STORM (dSTORM) since it does not use activator fluorophores ${ }^{7}$. A third class of superresolution microscopy techniques based on structured illumination was introduced in $2000^{8}$. Linear structured illumination microscopy (SIM) is, however, limited to a twofold increase in resolution, unlike STED and SML techniques.

In SLM based microscopy, the super-resolution is achieved by exploiting the fact that diffraction limits resolution, but not necessarily the localization precision of fluorophores. Specialized fluorophores that exhibit both a fluorescing (on) and a non-fluorescing (off) state are used to image only sparse subsets of the fluorophores simultaneously. The key requirement is that the active fluorophores are sparse enough to be identified as single emitters, meaning that they have to be separated by more than the diffraction limit. Having the prior information that the signals

Nanoimaging and Nanospectroscopy V, edited by Prabhat Verma, Alexander Egner, Proc. of SPIE

Vol. 10350, 103500W - (c) The Authors. Published under a Creative Commons Attribution

CC-BY 3.0 License · doi: 10.1117/12.2273902

Proc. of SPIE Vol. 10350 103500W-1 
(photons) come from the single emitter (single fluorophore molecule), the two-dimensional Gaussian profile can be fitted with high accuracy to determine the center location of the fluorophore. The localization precisions of the center position is influenced by the signal to noise ratio and with an increase of photons number the localization precision of $10 \mathrm{~nm}$ or better can be obtained.

Since the discovery of optical nanoscopy, the field has emerged rapidly and optical nanoscopes are slowly making inroads in mainstream biology and life sciences. However, present day optical nanoscopy still possess several challenges, with the most prominent being: costly and complex microscopes, lack of multi-modality, low temporal resolution, need of special fluorophores, and low-throughput.

There is a growing interest for high-throughput imaging in biological research ${ }^{9}$. High-throughput diffraction limited fluorescence microscopy is used in biological research, partly motivated by the pharmaceutical industry. Highthroughput can refer to many things: high-speed imaging, parallel imaging, increased field-of-view (f-o-v) or multiplexing. Super-resolution microscopy, however, poses new challenges when moving towards high-throughput imaging. Increased temporal resolution has seen much interest, with SIM performing well, albeit at limited resolution ${ }^{10}$. STED can also achieve very high temporal resolution, when performing imaging over small field-of-views ${ }^{11}$. In 2014, $d S T O R M$ was also performed at video-rate acquisition by employing specialized fitting algorithms for sCMOS cameras in combination with multi-emitter fitting algorithms ${ }^{12}$.

Super-resolution optical microscopy has also been moving towards high throughput by imaging an increased fo-v. STED is a confocal based point scanning technique and therefore inherently slow when imaging large areas. On the other hand, the techniques like SML microscopy and SIM are wide field techniques. In SML microscopy techniques, such as $d S T O R M$, the sample is commonly illuminated either by the evanescent field generated using a total internal reflection fluorescence (TIRF) objective lens, or a highly inclined thin illumination (HiLo) from the same type of lens. A TIRF lens with high N.A. (e.g 1.4) is required to illuminate the sample with an angle of incidence greater than the critical angle of incidence to generate evanescent field at the interface. The high N.A. TIRF lens also comes with high magnification (typically 60-100X) that limits the f-o-v down to around $100 \times 100 \mu \mathrm{m}^{2}$. Despite rather small f-o-v, simultaneous multi-color imaging has been achieved in various ways ${ }^{13-15}$, reducing collection time significantly.

In this paper, we show TIRF and super-resolution imaging using a photonic chip waveguide platform that is ideally suited for high-throughput TIRF and super-resolution imaging ${ }^{16}$. Waveguide structures are today commonly employed in e.g. sensing applications ${ }^{17}$, Raman spectroscopy ${ }^{18}$ and optical trapping ${ }^{19,20}$. Integrated photonic circuit enables easy beam shaping, and is well suited for integration with e.g. microfluidic devices. The key aspect of the waveguide platform is that the guided light will generate an evanescent field at the surface of the waveguide structure. As the evanescent field decays over 100-200 nm, it offers intrinsic optical sectioning. The sample is placed directly on top of the waveguide surface and is illuminated by the evanescent field of the waveguide (Fig. 1a). By using highrefractive index materials for the waveguide structures and by confining the light tightly inside a thin waveguide (100$200 \mathrm{~nm}$ thick) the light in the evanescent field can be intensified, enabling fluorophore blinking, which is a key requirement for $d$ STORM. Traditionally, super-resolution imaging is performed with advanced microscopes to excite and collect the fluorescence. In the proposed set-up, the photonic circuits are used both to hold and to excite the sample, whereas the signal is collected using a simple microscope. The photonic chip-based system has many important advantages. It decouples the excitation and the collection light paths. As discussed above, in conventional $d S T O R M$ a TIRF objective lens is commonly used both to excite the fluorophores and to collect the emitted fluorescence, therefore limiting the f-o-v. Instead of using the objective lens to both generate evanescent field excitation and to collect fluorescence, the waveguide platform is used to produce evanescent field illumination over the whole structure. The illumination area can therefore be very large, as it is only limited by the waveguide size, unlike TIRF objectives. By using a low magnification lens for collection, large areas can be imaged. The increased depth of field of lower magnification lenses is not a problem, as waveguides give intrinsic optical sectioning. Additionally, the photonic chips allow easy integration with other integrated optical functions such as e.g. optical trapping ${ }^{19,20}$ and Raman spectroscopy ${ }^{21}$. Finally, the waveguide chips can be made cost-effective by mass-production.

Our aim was to investigate the potential of the waveguide platform for large f-o-v $d S T O R M$ imaging. Using the photonic chip based TIRF microscope, we image the plasma membrane of liver sinusoidal endothelial cells (LSECs) and trophoblasts (HTR-8 cells) with dSTORM. We were able to image the LSEC fenestrations, transmembrane holes of 50$150 \mathrm{~nm}$ in diameter, morphological characteristic of these cells, demonstrating a resolution of around $70 \mathrm{~nm}$. dSTORM imaging was performed over modest $\mathrm{f}-\mathrm{o}-\mathrm{v}$ (using 60x/1.2 N.A. WI objective lens), but we also present a TIRF image of LSEC microtubulin over $500 \mu \mathrm{m} \times 500 \mu \mathrm{m}(25 \mathrm{x} / 0.8 \mathrm{~N}$.A. WI/OI objective lens). This clearly illustrates that the waveguide platform is ideally suited for large area TIRF excitation, a prerequisite for large f-o-v $d S T O R M$ imaging. 


\section{METHODS}

\subsection{CHIP-BASED NANOSCOPE}

The experimental set-up of the chip-based optical nanoscope is shown in Fig. 1b. In this work, we used an Olympus compound microscope for collection. A Cobolt Flamenco $660 \mathrm{~nm}$ laser and an Oxxius $488 \mathrm{~nm}$ laser were used for the excitation. The laser beams were expanded to slightly overfill the coupling objective, coupling into a single-mode fiber. Light is coupled into to the planar waveguides from the single mode fiber by allowing the cleaved fiber tip to almost touch the end facet of the waveguide. The portion of light that is coupled into the waveguide will be guided in discrete modes along the full stretch of the waveguide. For wide waveguides, a large number of modes will populate the structure in multi-mode interference patterns. The effect is rather devastating on the homogeity of the evanescent field, resulting in a non-uniform excitation of the fluorophores. To compensate for this, we scramble the modes using a piezo scanning translation stage. The input fiber is shifted laterally along the waveguide facet using the piezo stage, resulting in a new subset of modes for every new position of the input fiber. Depending on the speed of the mode scrambling the average of a set frames will give a much more homogenous excitation. Additionally, the piezo stage is also used for optimizing the coupling on to the planar waveguide initializing the experiment. Chips were held using a vacuum stage on top of an xyzstage. The coupling and sample stages were mounted on a long travel distance stage for sample translation. The emitted fluorescence is collected using a 60x/1.2NA WI objective lens for small f-o-v or a 25x/0.85 NA WI/OI objective lens for large f-o-v. The collected signal is passed through a 664 LP filter and 692/40 emission filter. For $488 \mathrm{~nm}$ excitation, a 488 LP filter and a 520/36 emission filter were used. An Olympus tube lens forms the image on an sCMOS camera (Hamamatsu ORCA Flash v2). TIRF imaging was done at $100 \mathrm{~mW}$ from the laser, passing through an ND 2.0 filter. The dSTORM images were captured using $550 \mathrm{~mW}$ power from the $660 \mathrm{~nm}$ laser. Typical camera exposure times for TIRF imaging is in the range of $100 \mathrm{~ms}$, while dSTORM imaging is acquired around $30 \mathrm{~ms}$, although individual adjustments are required for the blinking ratio of the given fluorophore/experiment.

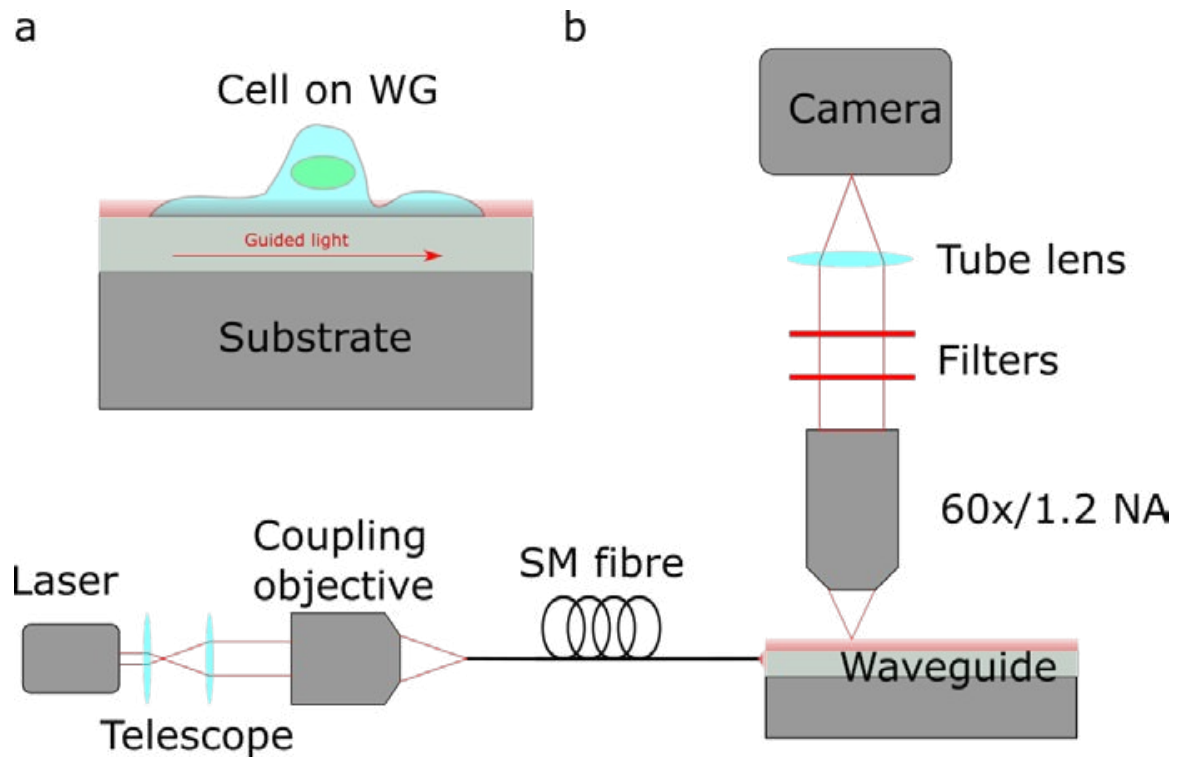

Figure 1: (a) Illustration of how the evanescent field can be used in TIRF imaging. The light is guided inside the waveguide, generating an evanescent field stretching approximately 100-150 nm out of the waveguide. The sample is placed in contact with the waveguide structure. (b) Schematic of the set-up, with fiber coupling into the waveguide and collection through a standard microscope. 


\subsection{SAMPLE PREPARATION}

The waveguides were fabricated using previously designed procedures ${ }^{22,23}$ The waveguides were cleaned before experiments for 10 minutes in $1 \%$ Hellmanex in deionized (DI) water at a $70^{\circ} \mathrm{C}$ hotplate. Following soaking, the waveguides were rinsed with DI water, iso-propanol and DI water again. A hollow rectangular PDMS frame was applied to form the sample chamber, containing the aqueous imaging buffer and to limit the cell attachment. The PDMS frames are shaped like small windows forming a sample chamber, open in the center outlining the imaging area. The thickness of the PDMS chambers is kept shallow $(130 \mu \mathrm{m})$ to not exceed the working distance of the microscope objectives. The waveguides surface was coated with human fibronectin $\left(50 \mathrm{\mu gml}^{-1}\right)$ for $10 \mathrm{~min}$ at RT prior to cell seeding. Isolated rat LSECs in RPMI 1640 were seeded on the waveguide and allowed attachment for $1 \mathrm{~h}$ at $37^{\circ} \mathrm{C}$ in incubator, followed by removal of non-adherent cells, and another $1 \mathrm{~h}$ for cytoplasm extension. Human placenta epithelial trophoblast cell line (HTR-8) were seeded on waveguides and incubated for 4 hours. Finally, the cells were washed with PBS and fixed with a solution of $4 \%$ paraformaldehyde in PBS and $0.02 \mathrm{M}$ sucrose (pH 7.2) for $30 \mathrm{~min}$.

The plasma membranes of both cell types were stained using Cell Mask Deep Red (CMDR), using the following protocol. The fixative was removed and the cell cultures rinsed with PBS 2 times for 5 minutes. A solution of $1 \%$ bovine serum albumin (BSA) in PBS was added to the cells for 30 minutes at RT to block unspecific binding sites prior to staining with CMDR. The cells were then permeabilized for 15 minutes at RT using $0.1 \%$ Triton-X in $1 \%$ BSA in PBS . The permeabilized cells were stained using a 1:1000 CMDR in PBS solution, incubating for 30 minutes and then rinsed with PBS. Microtubules were stained using an anti-tubulin-Alexa Fluor ${ }^{\circledR} 488$ antibody (1:100 dilution in 5\% BSA for 60 minutes). Once the samples were labeled, the chamber was sealed with a cover slip. A rectangular piece of black PDMS, with a transparent thin bottom was placed on top of the waveguide to block stray light. The photonic chip was then mounted on the vacuum holder and the laser light coupled into the waveguide structure. The dSTORM imaging is carried out in a traditional buffer containing an enzymatic oxygen scavenging system (glucose oxidase and catalase in glucose) and a reducing thiol (100 mM MEA) in $\mathrm{PBS}^{24}$.

\subsection{WAVEGUIDE FABRICATION}

$\mathrm{Si}_{3} \mathrm{~N}_{4}$ waveguide were fabricated by standard CMOS fabrications processes. A $2 \mu \mathrm{m}$ silica layer was grown thermally on the silicon wafers as a substrate. $\mathrm{Si}_{3} \mathrm{~N}_{4}$ layer of $150 \mathrm{~nm}$ was then deposited using low-pressure chemical vapor deposition (LPCVD) at $800^{\circ} \mathrm{C}$. Waveguide structuring was done by standard photolithography, followed by reactive ion etching to fabricate strip structures. The masking photoresist was then removed. A $1.5 \mu \mathrm{m}$ top layer of silica was then grown using PECVD at $300{ }^{\circ} \mathrm{C}$. The top cladding prevents sample interactions with the waveguides outside the desired areas. Imaging areas were then opened up with RIE etching, exposing the waveguide to the sample. The resulting structures were 150 $\mathrm{nm}$ thick $\mathrm{Si}_{3} \mathrm{~N}_{4}$ waveguides of different width, with only the imaging areas uncladded. Waveguide widths ranged from $40 \mu \mathrm{m}$ to $500 \mu \mathrm{m}$.

\section{RESULTS AND DISCUSSIONS}

The liver sinusoidal endothelial cells (LSECs) form the wall of the hepatic capillary blood vessels, the sinusoids. Their functions are related to trafficking of molecules and cells between the liver parenchyma and the blood, scavenging of potential dangerous macromolecules from the blood, liver immunity, and liver aging ${ }^{25}$. LSECs have a very distinct morphological characteristic represented by nano-size transmembrane pores, or fenestrations, organized in sieve plates. These fenestrations have a diameter of 50-200 $\mathrm{nm}$ and allow solutes and colloids to pass through, but blocks larger particles such dietary lipids like chylomicrons. The fenestrated sieve plates are generally found in the extensive, attenuated parts of the cytoplasm, where the thickness of the cell is approximately 150 to $170 \mathrm{~nm}^{26}$. Because of the thickness of the cytoplasm and the size of the fenestrations, TIRF dSTORM is ideal for imaging LSECs. Sufficiently high intensities $\left(0.5-10 \mathrm{KW} / \mathrm{cm}^{2}\right)$ for fluorophore blinking are achieved in the evanescent field by using high refractive index materials in the waveguides. Using optical waveguides, we performed dSTORM imaging of the LSECs'. Figure 2 presents diffraction limited and super-resolved images of the plasma membrane of LSECs. We were able to visualize fenestrations in the plasma membrane of around $70 \mathrm{~nm}$ size, as seen from the line plot in Figure 3. 

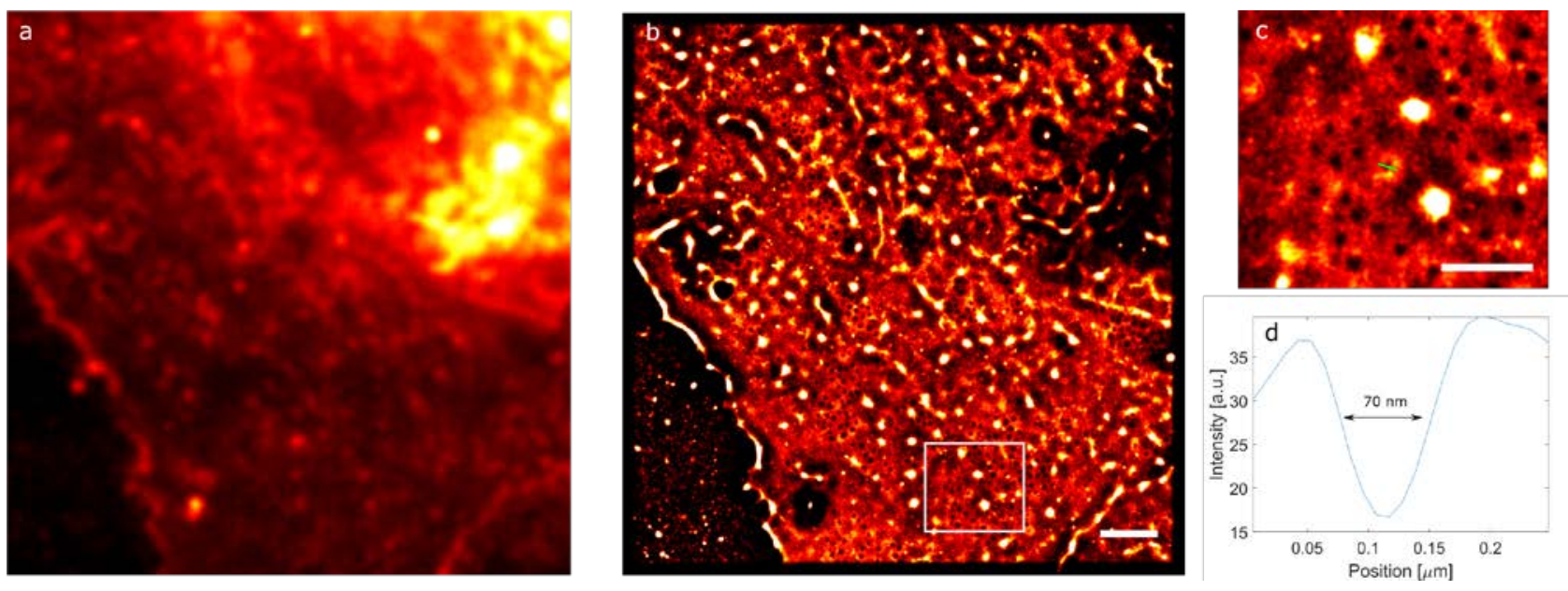

Figure 2: (a) TIRF image of LSEC. (b) dSTORM image of same region of LSEC. Multiple fenestrations are clearly visible in the super-resolved image (examples are found e.g. inside the white rectangle). The images were captured using a 60x/1.2 NA water immersion objective lens. Scale bar is $2 \mu \mathrm{m}$. (c) Enlarged area from figure (b) (marked by the white rectangle). Scale bar is $1 \mu \mathrm{m}$. (d) Line plot along the green line in figure (c), demonstrating a resolution of $70 \mathrm{~nm}$ for the fenestration diameter.

To further highlight the potential of the technique, we stained the plasma membrane of HTR-8 cells with Cell Mask Deep Red. In Figure 3, we show both the diffraction limited (a) and dSTORM (b) images of the HTR-8 plasma membrane. HTR-8 cells form the outer layer of the placenta and express filopodia, which are actin-rich plasma membrane protrusions with roles in sensing, migration and cell-cell interaction ${ }^{27}$, both imaging methods show the filopodia, but $d$ STORM imaging gives a higher contrast and increased resolution (220 nm) as compared to the diffraction limited TIRF image (450 nm) (Figure 3).
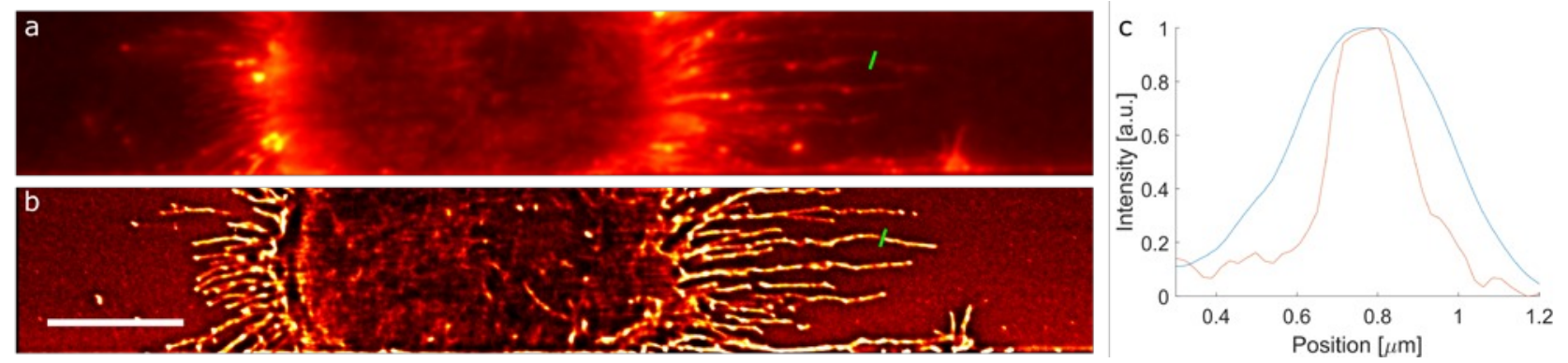

Figure 3: a) TIRF image of the plasma membrane of HDR-8 cells. b) $d$ STORM image of HTR-8 cells. The contrast is greatly improved, and the resolution enhancement is best visible in the filopodia. The images were captured using a $60 \mathrm{x} / 1.2$ NA water immersion objective lens. Scale bar is $10 \mu \mathrm{m}$. (c) Line plot along the filopodium marked with green line in figure (a and b), with FWHM of $450 \mathrm{~nm}$ for TIRF and 220 for $d$ STORM.

One of the main advantages for chip-based microscopy is the decoupling of the excitation and emission light paths. This enables us to acquire images using both high and low NA objectives, and still keep the benefit of TIRF illumination. Chip based microscopy therefore offers a choice between resolution and field of view, but without compromising the advantages of having a perfect TIRF illumination. In Figure 4, we present a $500 \mu \mathrm{m}$ x $500 \mu \mathrm{m}$ TIRF image of LSEC microtubules is presented. The image was taken with a $25 \mathrm{x} / 0.85 \mathrm{NA}$ objective lens and a $500 \mu \mathrm{m}$ wide waveguide was used for excitation. The extra-ordinary large f-o-v TIRF images enables visualization of over 100 LSECs within a single $\mathrm{f}-\mathrm{o}-\mathrm{v}$. Achieving large area TIRF excitation is the first step towards large f-o-v dSTORM imaging. Additionally, the complexity of the chip based optical setup is reduced when compared to traditional TIRF setup, as the need to steer the light precisely in the back focal plane of the TIRF objective lens is gone. Further more, multiplexing of wavelengths is 
straightforward. In traditional TIRF microscopy, the critical angle in the TIRF objective must be adjusted for each excitation wavelength, whereas optical waveguide provides pre-aligned geometry for the generation of the evanescent field for any wavelength of the light coupled and guided inside the optical waveguide. The high refractive index material allows tight confinement of the light inside the waveguide and by making thin waveguide $(150 \mathrm{~nm})$ a high intensity in the evanescent field can be generated that allows for efficient photoswitching of single molecules, even for relatively wide structures (Fig. 2-3). With optimized waveguide structures, it should be possible to perform $d$ STORM over areas equal to that in Figure 2. The system is ideal for imaging thin samples, or samples with defined structures within the reach of the evanescent field such as focal adhesion points, membrane fenestration (see Fig. 2), membrane nuclear pores and membrane trafficking.
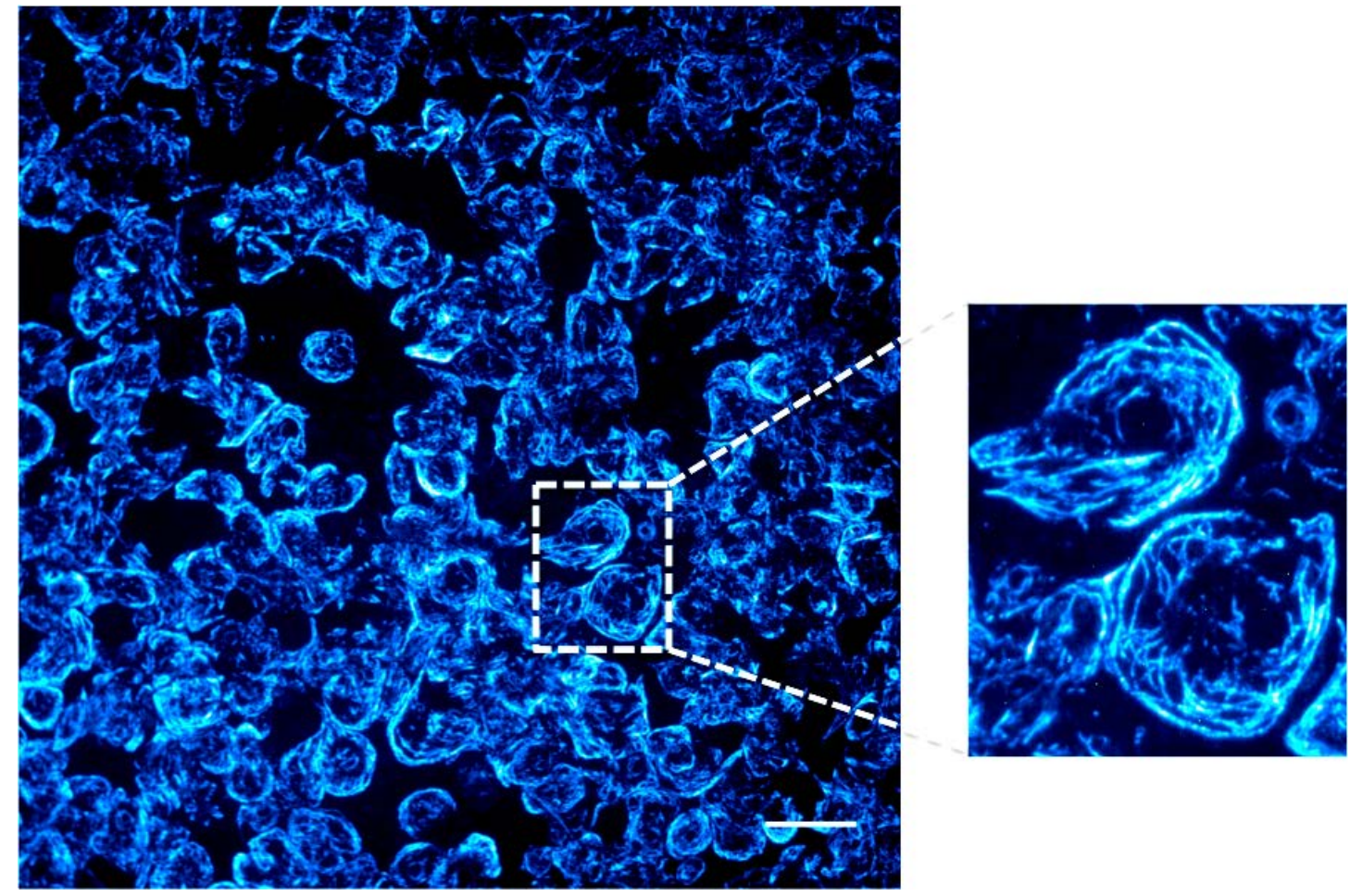

Figure 4: Large field of view TIRF image of LSEC microtubules stained with an anti-tubulin-Alexa Fluor ${ }^{\circledR} 488$ antibody. The image was captured with a $25 \mathrm{x} / 0.85 \mathrm{NA}$ objective lens and with a $500 \mu \mathrm{m}$ wide waveguide. Scale bar is $50 \mu \mathrm{m}$. The white dotted box shows the zoomed image of selected region.

\section{CONCLUSION}

We have demonstrated that high refractive index contrast waveguides $\left(\mathrm{Si}_{3} \mathrm{~N}_{4}\right)$ provide high intensity in the evanescent field, enabling single molecule localization microscopy based on SSTORM technique. Optical waveguides both hold the cells (LSEC and HTR-8) and excite the through the evanescent field. The separation of excitation and collection light paths provided by the optical waveguide platform opens up opportunities for large area TIRF excitation, a prerequisite for large f-o-v TIRF $d$ STORM imaging. The light coupled inside the waveguide is guided in a pre-defined path, generating an evanescent field all along the length of the waveguide. A large $\mathrm{f}-\mathrm{o}-\mathrm{v}$ can be achieved by increasing the width of the waveguide. We have presented very large $\mathrm{f}-\mathrm{o}-\mathrm{v}(500 \mu \mathrm{m} \times 500 \mu \mathrm{m})$ TIRF imaging of microtubulin in LSECs, showing that excitation in wide waveguides works well for imaging. The waveguide platform is thus a promising tool towards high throughput optical nanoscopy. 


\section{ACKNOWLEDGEMENTS}

Authors acknowledge assistance from Rajwinder Singh and Purusotam Basnet for their help with the preparation and the supply of HTR-8 cells. Authors acknowledge Jean-Claude Tinguely for his assistance with the optical waveguides. This work was supported by the European Research Council (grant no. 336716 to B.S.A.) and UiT, The Arctic University of Norway (Tematiske Satsinger to B.S.A.).

\section{References}

1. Ishikawa-Ankerhold, H. C., Ankerhold, R. \& Drummen, G. P. C. Advanced Fluorescence Microscopy Techniques-FRAP, FLIP, FLAP, FRET and FLIM. Molecules 17, 4047-4132 (2012).

2. Abbe, E. Beiträge zur Theorie des Mikroskops und der mikroskopischen Wahrnehmung. Arch. Für Mikrosk. Anat. 9, 413418 (1873).

3. Hell, S. \& Wichmann, J. Breaking the Diffraction Resolution Limit by Stimulated-Emission - Stimulated-Emission-

Depletion Fluorescence Microscopy. Opt. Lett. 19, 780-782 (1994).

4. Betzig, E. et al. Imaging intracellular fluorescent proteins at nanometer resolution. Science 313, 1642-1645 (2006).

5. Hess, S. T., Girirajan, T. P. K. \& Mason, M. D. Ultra-high resolution imaging by fluorescence photoactivation localization microscopy. Biophys. J. 91, 4258-4272 (2006).

6. Rust, M. J., Bates, M. \& Zhuang, X. Sub-diffraction-limit imaging by stochastic optical reconstruction microscopy (STORM). Nat. Methods 3, 793-796 (2006).

7. Heilemann, M. et al. Subdiffraction-Resolution Fluorescence Imaging with Conventional Fluorescent Probes. Angew. Chem. Int. Ed. 47, 6172-6176 (2008).

8. $\quad$ Gustafsson, M. G. L. Surpassing the lateral resolution limit by a factor of two using structured illumination microscopy. $J$. Microsc. 198, 82-87 (2000).

9. $\quad$ Pepperkok, R. \& Ellenberg, J. High-throughput fluorescence microscopy for systems biology. Nat. Rev. Mol. Cell Biol. 7, 690-696 (2006).

10. Kner, P., Chhun, B. B., Griffis, E. R., Winoto, L. \& Gustafsson, M. G. L. Super-resolution video microscopy of live cells by structured illumination. Nat. Methods 6, 339-342 (2009).

11. Westphal, V. et al. Video-Rate Far-Field Optical Nanoscopy Dissects Synaptic Vesicle Movement. Science 320, 246-249

(2008).

12. Huang, F. et al. Video-rate nanoscopy using sCMOS camera-specific single-molecule localization algorithms. Nat. Methods 10, 653-658 (2013).

13. Bates, M., Huang, B., Dempsey, G. T. \& Zhuang, X. Multicolor Super-Resolution Imaging with Photo-Switchable Fluorescent Probes. Science 317, 1749-1753 (2007).

14. Lampe, A., Haucke, V., Sigrist, S. J., Heilemann, M. \& Schmoranzer, J. Multi-colour direct STORM with red emitting carbocyanines. Biol. Cell 104, 229-237 (2012).

15. Zhang, Z., Kenny, S. J., Hauser, M., Li, W. \& Xu, K. Ultrahigh-throughput single-molecule spectroscopy and spectrally resolved super-resolution microscopy. Nat. Methods 12, 935-938 (2015).

16. Diekmann, R. et al. Chip-based wide field-of-view nanoscopy. Nat. Photonics 11, 322-328 (2017).

17. Fan, X. et al. Sensitive optical biosensors for unlabeled targets: A review. Anal. Chim. Acta 620, 8-26 (2008).

18. Dhakal, A. Nanophotonic Waveguide Enhanced Raman Spectroscopy. (2016).

19. Ahluwalia, B. S., Helle, Ø. I. \& Hellesø, O. G. Rib waveguides for trapping and transport of particles. Opt. Express 24, 4477 (2016).

20. Helle, Ø. I., Ahluwalia, B. S. \& Hellesø, O. G. Optical transport, lifting and trapping of micro-particles by planar waveguides. Opt. Express 23, 6601 (2015).

21. Dhakal, A. et al. Evanescent excitation and collection of spontaneous Raman spectra using silicon nitride nanophotonic waveguides. Opt. Lett. 39, 4025-4028 (2014).

22. Ahluwalia, B. S. et al. Fabrication of Submicrometer High Refractive Index Tantalum Pentoxide Waveguides for Optical Propulsion of Microparticles. IEEE Photonics Technol. Lett. 21, 1408-1410 (2009).

23. Prieto, F. et al. An integrated optical interferometric nanodevice based on silicon technology for biosensor applications. Nanotechnology 14, 907 (2003).

24. van de Linde, S. et al. Direct stochastic optical reconstruction microscopy with standard fluorescent probes. Nat. Protoc. 6, 991-1009 (2011).

25. McLean, A. J. et al. Age-related pseudocapillarization of the human liver. J. Pathol. 200, 112-117 (2003).

26. Wisse, E. et al. Structure and function of sinusoidal lining cells in the liver. Toxicol. Pathol. 24, 100-111 (1996).

27. Mattila, P. K. \& Lappalainen, P. Filopodia: molecular architecture and cellular functions. Nat. Rev. Mol. Cell Biol. 9, 446454 (2008). 